\title{
openheart Comparison of NICE and ESC proposed strategies on new onset chest pain and the contemporary clinical utility of pretest probability risk score
}

\author{
Alexandros Papachristidis (D) , ${ }^{1,2}$ George Frederick Vaughan, ${ }^{3}$ Sarah J Denny, ${ }^{4}$ \\ Tamim Akbari, ${ }^{1}$ Edith Avornyo, ${ }^{4}$ Tracey Griffiths, ${ }^{1}$ Emma Saunders, ${ }^{1}$ \\ Jonathan Byrne, ${ }^{1}$ Mark J Monaghan, ${ }^{1,2}$ Khaled Al Fakih ${ }^{4}$
}

To cite: Papachristidis A, Vaughan GF, Denny SJ, et al. Comparison of NICE and ESC proposed strategies on new onset chest pain and the contemporary clinical utility of pretest probability risk score. Open Heart 2020;7:e001081. doi:10.1136/ openhrt-2019-001081

Received 4 May 2019 Revised 2 December 2019 Accepted 17 February 2020

A Check for updates

(C) Author(s) (or their employer(s)) 2020. Re-use permitted under CC BY-NC. No commercial re-use. See rights and permissions. Published by BMJ.

${ }^{1}$ Cardiology, King's College Hospital NHS Foundation Trust, London, UK

${ }^{2}$ Cardiovascular Division, King's College London, London, United Kingdom

${ }^{3}$ GKT School of Medical Education, King's College London, London, UK

${ }^{4}$ Cardiology, Lewisham and Greenwich NHS Trust, London, UK

Correspondence to Dr Alexandros Papachristidis; alexandros.papachristidis@ nhs.net

\section{ABSTRACT}

Aims Patients with de novo chest pain are usually investigated non-invasively. The new UK-National Institute for Health and Care Excellence (NICE) guidelines recommend CT coronary angiography (CTCA) for all patients, while European Society of Cardiology (ESC) recommends functional tests. We sought to compare the clinical utility and perform a cost analysis of these recommendations in two UK centres with different primary investigative strategies.

Methods-results We compared two groups of patients, group $A(n=667)$ and group $B(n=654)$, with new onset chest pain in two neighbouring National Health Service hospitals, each primarily following either ESC (group A) or NICE (group B) guidance. We assessed the clinical utility of each strategy, including progression to invasive coronary angiography (ICA) and revascularisation. We present a retrospective cost analysis in the context of UK tariff for stress echo (£176), CTCA (£220) and ICA (£1001). Finally, we sought to identify predictors of revascularisation in the whole population.

Baseline characteristics in both groups were similar. The progression to ICA was comparable $(9.9 \%$ vs $12.0 \%$, $\mathrm{p}=0.377$ ), with similar requirement for revascularisation ( $4.0 \%$ vs $5.0 \%$.; $p=0.532$ ). The average cost of investigations per investigated patient was lower in group A (£279.66 vs £325.77), saving £46.11 per patient. The ESC recommended risk score (RS) was found to be the only predictor of revascularisation (OR $1.05,95 \% \mathrm{Cl} 1.04$ to $1.06 ; \mathrm{p}<0.001$ ).

Conclusion Both NICE and ESC-proposed strategies led to similar rates of ICA and need for revascularisation in discrete, but similar groups of patients. The SE-first approach had a lower overall cost by $£ 46.11$ per patient, and the ESC RS was the only variable correlated to revascularisation.

\section{INTRODUCTION}

The recent update of the UK National Institute of Health and Care Excellence (NICE) guidelines (2016) on the investigation of patients with chest pain recommends the use of CT coronary angiography (CTCA) key questions

What is already known about this subject?

- National Institute for Health and Clinical Excellence (NICE) has dramatically changed its guideline on stable chest pain aiming to find a more cost-effective strategy. It no longer recommends the use of pretest probability risk scores and it has removed functional tests, such as stress echocardiography, as first-line investigation in patients with new onset stable chest pain. It recommends using CT coronary angiography in the majority of patients. However, European Society of Echocardiography (ESC-2013) still suggest functional tests as the initial investigation. The change in NICE guidelines has unmasked the lack of CT facilities in many Trusts across the country and poses some uncertainty to clinicians who still use the ESC guidelines.

What does this study add?

- This study shows that following either NICE or ESC guidelines in two discrete but similar populations the progression rates to invasive coronary angiography and revascularisation are similar, but the stress echo-first strategy can reduce the average cost per investigated patient by £46.11. In addition, the pretest probability risk score, which has been removed from the UK NICE guidelines, seems to be the only predictor of revascularisation.

How might this impact on clinical practice?

- Following the publication of new NICE guidelines on stable chest pain, many cardiology departments face the challenge to downsize their well-established functional imaging services and expand their cardiac CT instead. Our study shows that stress echocardiography can still be used as an initial investigation for stable chest pain, and healthcare providers may follow either strategy, considering local service availability, expertise, local tariffs and budget.

as a first-line investigation in patients with new onset stable chest pain. ${ }^{1}$ NICE no longer recommend the use of their pretest 
probability (PTP) risk scores (RS), because it was found to significantly overestimate the PTP of coronary artery disease (CAD). They now recommend a clinical judgement on typicality of chest pain, followed by CTCA for patients with atypical and typical chest pain and also for patients with non-cardiac chest pain if they have ischaemic changes on the ECG. If the CTCA is non-diagnostic or demonstrates $\mathrm{CAD}$ of uncertain functional significance, they recommend functional testing.

The European Society of Cardiology (ESC) guidelines on chest pain of recent onset $(2013)^{2}$ recommend the use of a PTP RS which is based on age, gender and typicality of chest pain. Their RS is based on a modified DiamondForrester method, ${ }^{3}$ which adjusted the likelihood of CAD to a contemporary population. They recommend that patients with an RS of $<15 \%$ are investigated for other causes of chest pain. Patients with an RS of $15 \%-65 \%$ and normal LV function are recommended to have a functional imaging test, and if not available, an ECG exercise treadmill test (ETT). Patients with an RS of 66\%-85\% or reduced ejection fraction (EF) on echo with atypical chest pain are recommended to undergo functional imaging testing, while patients with typical chest pain and reduced $\mathrm{EF}$ are recommended to undergo invasive coronary angiography (ICA). CTCA is only recommended as an alternative to functional testing in patients with an RS of $15 \%-50 \%$.

The aim of this study is to compare the new NICE guidelines with the ESC guidelines for new onset stable chest pain in terms of progression to ICA and need for revascularisation. In addition, we sought to identify the most clinically effective and cost-effective pathway for patients with low/intermediate $(15 \%-50 \%)$ and intermediate/high ( $\geq 50 \%)$ PTP RS. The ultimate goal is to reduce the number of patients with non-obstructive $\mathrm{CAD}$ referred for ICA, which has been reported to be $62.4 \%$ in a registry from the USA. ${ }^{4}$ Finally, we explored the correlation of demographic and clinical variables, including the PTS RS, with coronary revascularisation.

\section{METHODS}

We investigated 1321 patients who were referred to the rapid access chest pain (RACP) clinics in two neighbouring National Health Service (NHS) hospitals in London, UK. Group A consisted of 667 consecutive patients who were referred from 1 January to 15 July 2017 and investigated at King's College Hospital, where the ESC guidelines on stable chest pain ${ }^{2}$ are followed. Group B consisted of 654 consecutive patients, who were referred from 1 January to 31 December 2017 and investigated at Lewisham University Hospital, where the updated NICE guidelines ${ }^{1}$ are followed.

\section{Stress echocardiography (SE)}

For the dobutamine SE, the standard protocol of $3 \mathrm{~min}$ stages was used, with incremental dobutamine doses of 5 , $10,20,30$ and $40 \mu \mathrm{g} / \mathrm{kg} / \mathrm{min}$. Intravenous atropine was given in bolus doses up to a maximum dose of $1200 \mu \mathrm{g}$ as necessary. The exercise SE used the Bruce treadmill exercise protocol. On both pharmacological and exercise SE, if the endocardium of two or more segments were not adequately visualised, an intravenous contrast agent was used. The echocardiography department's recommended policy is for ischaemia limited to apical segments only to be considered as suggestive of microvascular disease requiring no further investigation. ${ }^{5}$ Patients with two or more segments of non-apical ischaemia were considered to have a positive stress test and based on clinician's discretion they were further referred for ICA.

\section{CT coronary angiography}

For the CTCA, patients were beta-blocked by the referring clinician (bisoprolol $5 \mathrm{mg}$ ) and/or intravenously with metoprolol $(5-10 \mathrm{mg})$ aiming to achieve a heart rate of $<60$ beats $/ \mathrm{min}$. $80 \mathrm{~mL}$ of ivoersol (optiray $350 \mathrm{mg} \mathrm{I} / \mathrm{ml}$, Covidien UK, Hampshire, UK), at a flow rate of $6 \mathrm{~mL} / \mathrm{s}$, followed by $100 \mathrm{~mL}$ of saline solution, was injected into an antecubital vein. Bolus tracking was used with a region of interest placed into the ascending aorta.

All CTCA were performed with a 64 slice LightSpeed VCT XTe GE scanner (GE Healthcare) using the commercially available protocol (SnapShot Pulse, GE healthcare) and the following scanning parameters: slice acquisition $64 \times 0.625 \mathrm{~mm}$, scan field of view Cardiac, Z-axis detector coverage $40 \mathrm{~mm}$, gantry rotation time of $350 \mathrm{~ms}$. Patients' size was visually judged for adapted tube voltage; $100 \mathrm{kV}$ was used for small patients and $120 \mathrm{kV}$ for all other patients. Prospective gating is the standard acquisition protocol and was used in $98 \%$ of patients. A retrospective gated scanning was used in the remaining $2 \%$. CTCA images were reconstructed with slice thickness of $0.625 \mathrm{~mm}$, on an external workstation (ADW 4.5, GE Healthcare). The cardiology department's recommended policy is for patients found to have less than $50 \%$ coronary artery stenosis to be managed medically, patients with $50 \%-70 \%$ stenosis and equivocal symptoms to be referred for SE for further assessment, and those with a coronary artery stenosis $>70 \%$ to proceed to invasive angiography.

\section{Data collection and analysis}

The demographic data, cardiovascular risk factors, the characteristics of pain (typical angina, atypical angina, non-anginal pain), left ventricular EF and the result of the functional test were recorded prospectively. The ESC PTP RS was calculated according to published guidelines. ${ }^{2}$ Data regarding second-line investigations and revascularisation were recorded retrospectively from hospitals' electronic patient records. The invasive coronary angiograms were reported by an experienced interventional cardiologist. The decision for revascularisation was made by the interventional cardiologist or by a multidisciplinary team consisting of cardiologists and cardiothoracic surgeons.

The patients in both groups A and B were divided into two subgroups based on their PTP RS: in subgroups A1 
and B1, patients with low-intermediate PTP RS (15\%$50 \%)$ were included, whereas in subgroups A2 and B2 patients with intermediate-high PTP RS $(>50 \%)$.

For the cost analysis, the patients who were referred directly for ICA were excluded, as their investigation and management were considered to be independent of the guidelines followed in the two centres (NICE vs ESC). The cost of ECG exercise test, cardiac perfusion MRI and nuclear myocardial perfusion imaging (MPI) was not taken into account as very few patients were investigated with those tests and additionally the availability of MRI and MPI differed significantly in the two institutions which would bias the results. The cost analysis was performed in the context of the UK tariff for stress echo (£176), CTCA (£220), and ICA (£1001).

Subsequently, we sought to investigate possible correlation of demographic and clinical variables with coronary revascularisation. Variables were tested using univariable log-regression analysis. Those with $\mathrm{p}<0.2$ were entered in a multivariable model. The goodness of fit of the model was tested with the Hosmer-Lemeshow test and was found to be adequate. A receiver operating characteristic (ROC) analysis was performed for prediction of revascularisation.

The statistical analysis was performed with IBM SPSS, V.25 (IBM Corporation Software Group). The normality of the distribution for continuous variables was tested using histograms. The Student's t-test for independent samples was used to compare mean values of continuous variables with normal distribution, and the nonparametric Mann-Whitney U test for non-normally distributed variables. The values are presented as a mean value \pm SD. Categorical variables were tested with $\chi^{2}$ test and Fisher's exact test as appropriate and are presented as absolute values and percentages.

This study is not classified as research under NHS Health Research Authority Guidance, and there was no need to apply for ethical approval or patient permission. The study was assessed as a service evaluation by both Hospitals' Audit Committees and the relevant permission was granted.

\section{RESULTS}

The baseline characteristics of our population are presented in table 1 . The mean age of the whole cohort was $56.3 \pm 12.6$ years. In total, 817 patients $(61.8 \%)$ were considered to warrant investigation for their symptoms. A higher proportion of patients were investigated in group $\mathrm{B}$ than in group A $(71.6 \%$ vs $52.3 \% \mathrm{p}<0.001)$, which may be explained by a higher PTP RS of the referred patients in group B (37.6 \pm 21.2 vs $34.6 \pm 20.8, \mathrm{p}=0.010)$. However, the PTP RS, other demographic data and the prevalence of risk factors were similar between patients who went on to be investigated in both groups as shown in table 1 , except for symptoms profile as more patients in group B were considered to have typical angina compared with group A. The patients whose symptoms were considered to be non-cardiac and were discharged without being referred for investigation were excluded from further analysis. The same applies to those who did not attend or declined investigations.

\section{Management-revascularisation}

The management of group A patients is shown in figure 1 . From 667 patients who were seen in the RACP clinic, 349 $(52.3 \%)$ were investigated for CAD:

i. The majority (212 patients, $60.8 \%$ ) were initially referred for a SE. Three of them $(0.9 \%)$ were further investigated with cardiac perfusion MRI and five (1.4\%) with CTCA (two with calcium score only), as $\mathrm{SE}$ was submaximal or considered inconclusive. One of the patients who was investigated with CTCA underwent ICA and was revascularised. Twenty-one patients $(6.0 \%)$ were referred for ICA following a positive SE and 10 were revascularized (2.9\%).

ii. Sixty-seven patients $(19.2 \%)$ were referred for a CTCA. Eleven $(3.2 \%)$ of them had calcium score only due to technical limitations/patient preference. Five of them $(1.4 \%)$ went on to have an SE to clarify the significance of CAD, with one ending up having ICA, but revascularisation was not deemed necessary. From the remaining 56 patients, $6(1.7 \%)$ proceeded to ICA and only 1 required revascularisation.

iii. Fifteen patients $(4.3 \%)$ had an exercise ECG test for initial assessment and two of them were referred for further investigations (one for $\mathrm{SE}$ and one for CTCA) with no need for ICA. Seven (2.0\%) patients were investigated initially with cardiac perfusion MRI and one went on to have ICA with no need for revascularisation.

iv. Forty-seven patients (13.5\%) were deemed high risk and referred directly for ICA and $1 / 3$ of them (16 patients; $4.6 \%$ of total) were revascularised.

In group B, 468 out of 654 patients $(71.6 \%)$ were investigated, with the remaining failing to attend for test or declined the investigations offered or deemed not to need investigation (figure 2).

i. Sixty-four patients $(13.7 \%)$ were deemed unsuitable for a 64 slice CT scan, predominantly due to asthma as the clinicians felt it inappropriate to give highdose beta-blockers. These patients presented with a heart rate $>65$ beats $/$ min or irregular rhythm despite moderate beta-blockade and the clinicians considered reasonable to refer them for SE. Seven of them $(1.5 \%)$ were then referred for ICA. Two patients $(0.4 \%)$ were revascularised.

ii. The vast majority of patients $(331 ; 70.7 \%)$ were investigated with CTCA and 17 of them, had CT calcium score only as the CTCA could not be performed because the heart rate was still high despite betablockade. Nineteen patients $(4.1 \%)$ were referred for a functional test because CTCA was inconclusive or could not be performed due to technical or patient limitations. Sixteen of them had SE (one proceeded to ICA but no revascularisation was needed), two had 
Table 1 Baseline characteristics, investigation tests and revascularisation in patients who went on to be investigated in both groups

\begin{tabular}{|c|c|c|c|c|c|c|c|}
\hline & $\begin{array}{l}\text { Group A } \\
\mathrm{n}=349\end{array}$ & $\%$ & $\begin{array}{l}\text { Group B } \\
n=468\end{array}$ & $\%$ & $P$ value & All & $\%$ \\
\hline Age & $57.2 \pm 12.2$ & & $56.9 \pm 10.9$ & & 0.697 & $57.0 \pm 11.5$ & \\
\hline Gender (male) & 174 & $49.9 \%$ & 240 & $51.3 \%$ & 0.687 & 414 & $50.7 \%$ \\
\hline Type of pain & & & & & 0.003 & & \\
\hline Typical anginal & 46 & $13.2 \%$ & 89 & $19.0 \%$ & & 135 & $16.5 \%$ \\
\hline Atypical anginal & 252 & $72.2 \%$ & 341 & $72.9 \%$ & & 593 & $72.6 \%$ \\
\hline Non-anginal & 51 & $14.6 \%$ & 38 & $8.1 \%$ & & 89 & $10.9 \%$ \\
\hline ESC Risk Score & $39.2 .6 \pm 21.3$ & & $41.12 \pm 21.2$ & & 0.214 & $36.1 \pm 21.0$ & \\
\hline ESC Risk Score Group & & & & & 0.194 & & \\
\hline Low $(<15 \%)$ & 44 & $12.6 \%$ & 36 & $7.7 \%$ & 0.121 & 80 & $9.8 \%$ \\
\hline Low-intermediate (15\%-50\%) & 215 & $61.6 \%$ & 303 & $64.7 \%$ & & 518 & $63.4 \%$ \\
\hline Intermediate-high (51\%-85\%) & 83 & $23.8 \%$ & 116 & $24.8 \%$ & & 199 & $24.4 \%$ \\
\hline High (>85\%) & 7 & $2.0 \%$ & 13 & $2.8 \%$ & & 20 & $2.4 \%$ \\
\hline Hypertension & 154 & $44.1 \%$ & 199 & $42.5 \%$ & 0.705 & 353 & $43.2 \%$ \\
\hline Hypercholesterolaemia & 216 & $61.9 \%$ & 264 & $56.4 \%$ & 0.142 & 480 & $58.8 \%$ \\
\hline Diabetes & 79 & $22.6 \%$ & 94 & $20.1 \%$ & 0.403 & 173 & $21.2 \%$ \\
\hline Family history & 149 & $42.7 \%$ & 184 & $39.3 \%$ & 0.370 & 333 & $40.8 \%$ \\
\hline Smoking & 80 & $22.9 \%$ & 88 & $18.8 \%$ & 0.168 & 168 & $20.6 \%$ \\
\hline Ejection fraction & 111 & $31.8 \%$ & 81 & $17.3 \%$ & 0.146 & 192 & $23.5 \%$ \\
\hline Normal & 93 & $26.6 \%$ & 75 & $16.0 \%$ & & 168 & $20.6 \%$ \\
\hline Mildly impaired & 12 & $3.4 \%$ & 6 & $1.3 \%$ & & 18 & $2.2 \%$ \\
\hline Moderately impaired & 5 & $1.4 \%$ & 0 & $0.0 \%$ & & 5 & $0.6 \%$ \\
\hline Severely impaired & 1 & $0.3 \%$ & 0 & $0.0 \%$ & & 1 & $0.1 \%$ \\
\hline Functional test & 243 & $69.6 \%$ & 93 & $19.9 \%$ & & 336 & $41.1 \%$ \\
\hline Stress echo & 218 & $62.5 \%$ & 81 & $17.3 \%$ & $<0.001$ & 299 & $36.6 \%$ \\
\hline Exercise treadmill test & 15 & $4.3 \%$ & 2 & $0.4 \%$ & $<0.001$ & 17 & $2.1 \%$ \\
\hline MPI & 0 & $0.0 \%$ & 6 & $1.3 \%$ & 0.034 & 6 & $0.7 \%$ \\
\hline CMR perfusion & 10 & $2.9 \%$ & 4 & $0.9 \%$ & 0.016 & 14 & $1.7 \%$ \\
\hline CTCA/CT Calcium Score only & $60 / 13$ & $17.2 \% / 3.7 \%$ & $313 / 18$ & $66.9 / 3.9 \%$ & $<0.001$ & & \\
\hline Calcium Score & $33.3 \pm 129.8$ & & $87.3 \pm 252.4$ & & 0.037 & & \\
\hline ICA & 77 & $22.1 \%$ & 95 & $20.3 \%$ & 0.608 & 172 & $21.1 \%$ \\
\hline $\mathrm{PCl}$ & 22 & $6.3 \%$ & 27 & $5.8 \%$ & 0.750 & 49 & $6.0 \%$ \\
\hline CABG & 6 & $1.7 \%$ & 14 & $3.0 \%$ & 0.244 & 20 & $2.4 \%$ \\
\hline Revascularisation & 28 & $8.0 \%$ & 41 & $8.8 \%$ & 0.708 & 69 & $8.4 \%$ \\
\hline
\end{tabular}

Bold values represent statistical significance or in other words $p$ value $<0.05$.

CABG, coronary artery bypass grafting; CMR, cardiac magnetic resonnance; CTCA, CT coronary angiography; ESC, European Society of Cardiology; ICA, invasive coronary angiography; MPI, myocardial perfusion imaging; PCI, percutaneous coronary intervention.

MRI (one proceeded to ICA with no revascularisation) and one patient had stress nuclear MPI. Fortyone patients $(8.8 \%)$ went on to have ICA directly after cardiac CT and 19 (4.1\%) were revascularised.

iii. Two patients $(0.4 \%)$ were referred for exercise ECG test and one of them proceeded to SE, which was normal. Two patients $(0.4 \%)$ were investigated initially with cardiac perfusion MRI (one of them was referred for ICA) and five (1.1\%) had stress nuclear
MPI as first-line investigation. None of the above patients was revascularised.

iv. Forty-four patients $(9.4 \%)$ were deemed high risk and were referred directly for ICA and $46 \%$ of them (20 patients; $4.3 \%$ of total) were revascularised.

The progression of investigated patients to ICA, excluding those with direct ICA referral, was comparable between the two groups $(9.9 \%$ vs $12.0 \%, \mathrm{p}=0.377)$, as was the rate of revascularisation $(4.0 \%$ vs $5.0 \%$; $\mathrm{p}=0.532$; 


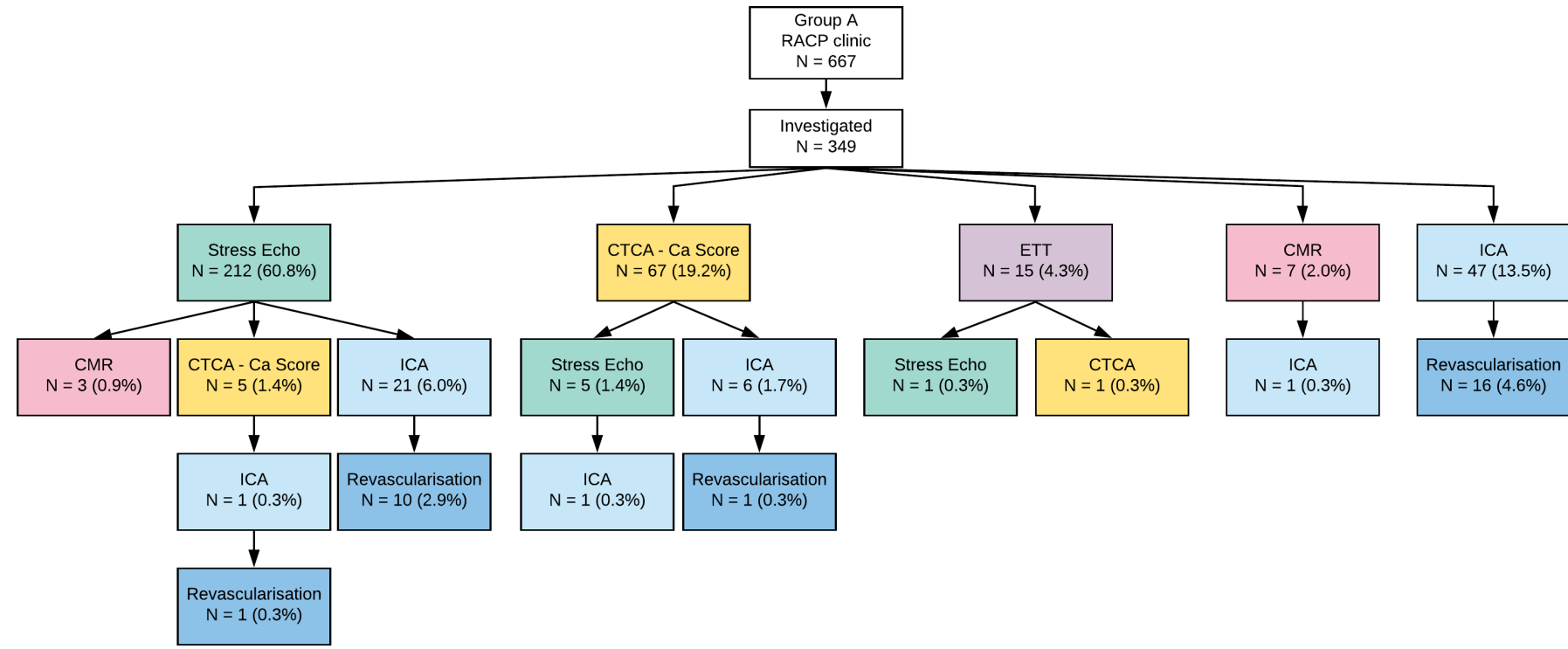

Figure 1 Flow chart showing the investigations and management of patients in group A. CMR, cardiac magnetic resonance; CTCA, CT coronary angiography; ETT, exercise treadmill test; ICA, invasive coronary angiography; MPI, myocardial perfusion imaging; RACP, rapid access chest pain.

table 2). By applying the standard UK tariff for SE, CTCA and ICA, the average cost per investigated individual was lower in group A by 446.11 ( 2279.66 vs 3325.77$)$.

\section{Subgroup analysis and cost analysis}

In patients with low-intermediate PTP RS (15\%-50\%) (table 3 ), there was a numerically lower rate of progression to ICA in group A1 compared with group B1 $(8.7 \%$ vs $12.6 \%, p=0.177$ ), but not statistically significant. Similarly, no difference between groups was observed for revascularisation $(2.6 \%$ vs $5.5 \%$; $\mathrm{p}=0.122)$. The average cost per investigated patient was considerably lower in group A1 compared with group B1 by $£ 69.54$.

In patients with intermediate-high PTP RS (>50\%), $18.5 \%$ proceeded to ICA after first-line investigation in group A2 compared with $14.6 \%$ in group $\mathrm{B} 2(\mathrm{p}=0.512$; table 2). The revascularisation rate was also similar between groups $(10.8 \%$ vs $5.2 \%$; $\mathrm{p}=0.187)$. The average per patient cost was slightly higher in group A2 compared with group B2 by £20.99. For the above comparisons, the patients who were referred directly for ICA were excluded.

\section{Variables associated with revascularisation}

Age, male gender, type of chest pain and the PTP RS were independently correlated to revascularisation in univariable log-regression analysis (table 4). The RS was then entered into a multivariable model along with hypertension, diabetes and smoking which were found to be correlated to revascularisation with $\mathrm{p}<0.2$ (table 4 ). Age, gender and type of pain were not included as they are components of the PTP RS. ESC RS was the only independent factor related to revascularisation in the multivariable analysis (OR 1.05, 95\% CI 1.04 to 1.06 ; $\mathrm{p}<0.001$; table 4).

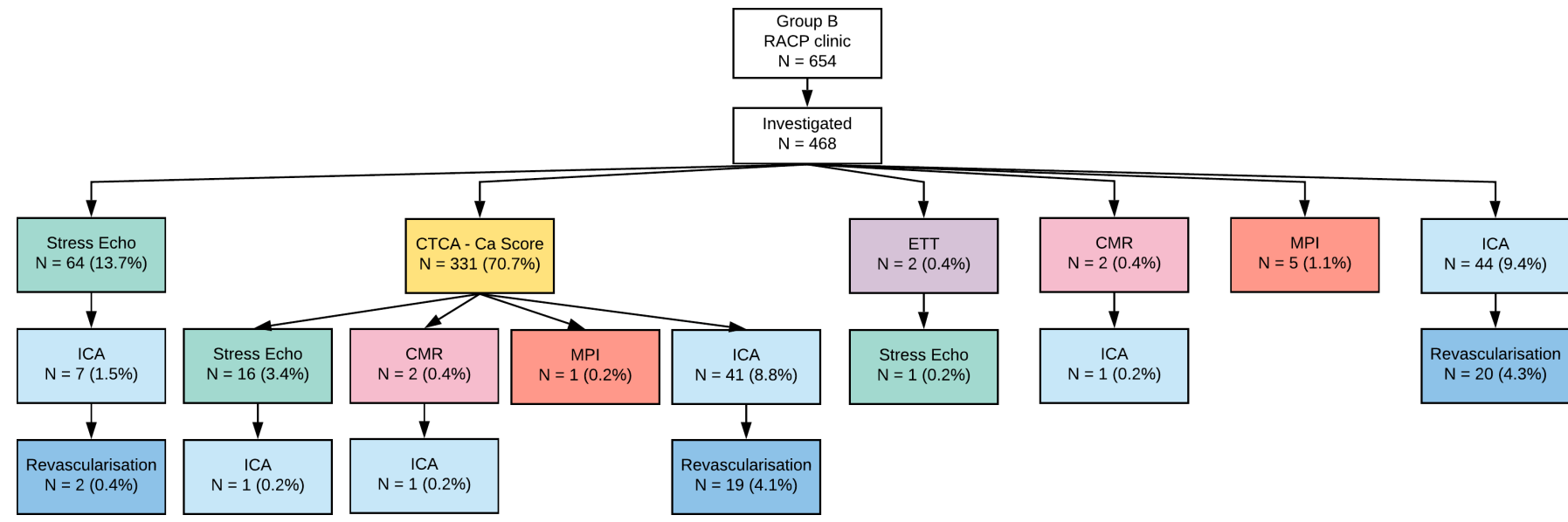

Figure 2 Flow chart showing the investigations and management of patients in group B. CMR, cardiac magnetic resonance; CTCA, CT coronary angiography; ETT, exercise treadmill test; ICA, invasive coronary angiography; MPI, myocardial perfusion imaging; RACP, rapid access chest pain. 
Table 2 Progression to invasive coronary angiography (ICA) and revascularisation after initial non-invasive testing in groups $\mathrm{A}$ and $\mathrm{B}$

\begin{tabular}{|c|c|c|c|c|c|c|c|}
\hline & $\begin{array}{l}\text { Group A } \\
\mathrm{n}=302\end{array}$ & $\%$ & $\begin{array}{l}\text { Group B } \\
n=424\end{array}$ & $\%$ & $P$ value & All & $\%$ \\
\hline ICA & 30 & $9.9 \%$ & 51 & $12.0 \%$ & 0.377 & 81 & $11.2 \%$ \\
\hline Revascularisation & 12 & $4.0 \%$ & 21 & $5.0 \%$ & 0.532 & 53 & $4.5 \%$ \\
\hline $\mathrm{PCl}$ & 10 & $3.3 \%$ & 14 & $3.3 \%$ & 0.994 & 24 & $3.3 \%$ \\
\hline CABG & 2 & $0.7 \%$ & 7 & $1.7 \%$ & 0.318 & 16 & $1.2 \%$ \\
\hline Total investigations cost & $£ 84458.0$ & & $£ 138127.0$ & & & $£ 222585.0$ & \\
\hline Average investigations cost & $£ 279.66$ & & $£ 325.77$ & & & $£ 306.59$ & \\
\hline
\end{tabular}

CABG, coronary artery bypass grafting; $\mathrm{PCl}$, percutaneous coronary intervention.

An ROC analysis was performed, and an ROC curve was plotted for PTP RS using revascularisation as a classification variable (figure 3 ). The area under the curve was 0.797 (95\% CI 0.743 to $0.851, \mathrm{p}<0.001)$. An RS cutoff value of 45.5 was found to have sensitivity $73.9 \%$ and specificity $70.4 \%$ for predicting revascularisation.

\section{DISCUSSION}

The main findings of our study are: (1) following either SE or CTCA as first-line investigation for new onset stable chest pain, the progression rates to ICA and the need for revascularisation are similar; (2) the average cost per investigated patient is lower (by £46.11) based on UK tariffs, when patients are initially investigated with SE; (3) in patients with low-intermediate PTP RS, the average investigation cost is considerably lower (by £69.54) if SE is selected as first-line investigation, but in patients with an intermediate-high RS it is slightly higher (by £20.99) with the same rate of progression to ICA and revascularisation in both subgroups, and (4) the ESC RS is the only independent predictor of revascularisation.

NICE have changed dramatically their recommendation on new onset stable chest pain, and they suggest the majority of patients to be investigated with an anatomical imaging test, namely CTCA. The functional tests, such as $\mathrm{SE}$, have been downgraded as second-line investigations in patients who cannot have a CTCA or when CTCA is inconclusive. ${ }^{1}$ There is a significant ongoing debate on anatomical versus functional tests as first-line investigations, ${ }^{67}$ and in our study, we explored how both strategies perform in a real-life population.

NICE suggest that investigating patients with new onset stable chest pain initially with CTCA can reduce the overall cost of investigations and CAD can be diagnosed more accurately. ${ }^{18}$ Our study suggests that both strategies (ie, CTCA vs SE as first-line investigations) lead to similar rates of ICA and need for revascularisation with a lower cost for SE-first approach. The clinical question of anatomical versus functional first-line test has been addressed in several studies. PROMISE (PROspective Multicenter Imaging Study for Evaluation of Chest Pain), a large outcome trial on 10003 patients, compared CTCA versus functional imaging tests (mostly stress nuclear MPI) in patients with new onset stable chest pain and intermediate risk, and found no difference in outcomes. ${ }^{9}$ There was low event rate of $3 \%$ in functional tests and $3.3 \%$ in CTCA arms. However, CTCA lead to a higher rate of ICA $(13.3 \%$ vs $5.1 \%)$ and revascularisation $(6.2 \%$ vs $3.2 \%$; $<<0.001)$.

SCOT-HEART (Scottish COmputed Tomography of the HEART Trial) is another major clinical trial, where patients were randomised to standard clinical evaluation (which was ETT) versus ETT plus CTCA. Four thousand and eighty patients completed a follow-up over a median

Table 3 Investigations, revascularisation rate and cost analysis in subgroups of low-intermediate (15\%-50\%) and intermediate-high (>50\%) risk score

\begin{tabular}{|c|c|c|c|c|c|c|}
\hline & PTP RS: 1 & & & PTP RS >5 & & \\
\hline & $\begin{array}{l}\text { Group A1 } \\
\mathrm{N}=195\end{array}$ & $\begin{array}{l}\text { Group B1 } \\
\mathrm{N}=293\end{array}$ & $P$ value & $\begin{array}{l}\text { Group A2 } \\
\mathrm{N}=65\end{array}$ & $\begin{array}{l}\text { Group B2 } \\
\mathrm{N}=96\end{array}$ & $P$ value \\
\hline Stress echo & $140(71.8 \%)$ & $48(16.4 \%)$ & $<0.001$ & $59(90.8 \%)$ & $28(29.2 \%)$ & $<0.001$ \\
\hline CTCA & 47 (24.1\%) & $241(82.3 \%)$ & $<0.001$ & $4(6.2 \%)$ & $61(63.5 \%)$ & $<0.001$ \\
\hline ICA & $17(8.7 \%)$ & $37(12.6 \%)$ & 0.177 & $12(18.5 \%)$ & $14(14.6 \%)$ & 0.512 \\
\hline Revascularisation & $5(2.6 \%)$ & $16(5.5 \%)$ & 0.122 & 7 (10.8\%) & $5(5.2 \%)$ & 0.187 \\
\hline Total cost & $£ 51997.0$ & $£ 98505.0$ & & $£ 23276.0$ & $£ 32362.0$ & \\
\hline Average cost & $£ 266.65$ & $£ 336.19$ & & $£ 358.09$ & $£ 337.10$ & \\
\hline
\end{tabular}

CTCA, CT coronary angiography; ICA, invasive coronary angiography; PTP RS, pretest probability risk score. 
Coronary artery disease

Table 4 Univariable and multivariable log-regression analysis of factors correlated with revascularisation

\begin{tabular}{|c|c|c|c|c|}
\hline \multirow[b]{2}{*}{ Variable } & \multicolumn{2}{|c|}{ Univariable analysis } & \multicolumn{2}{|c|}{ Multivariable analysis } \\
\hline & OR $(95 \% \mathrm{Cl})$ & $P$ value & OR $(95 \% \mathrm{Cl})$ & $P$ value \\
\hline Age & 1.05 (1.03 to 1.07$)$ & $<0.001$ & & \\
\hline Gender (male) & 2.76 (1.60 to 4.77 ) & $<0.001$ & & \\
\hline Type of pain & & $<0.001$ & & \\
\hline Non-anginal & 1.00 (Reference) & & & \\
\hline Atypical & 2.56 (0.60 to 10.87$)$ & 0.202 & & \\
\hline Typical & 14.64 (3.42 to 62.72$)$ & $<0.001$ & & \\
\hline Risk score & 1.05 (1.04 to 1.06$)$ & $<0.001$ & 1.05 (1.04 to 1.06$)$ & $<0.001$ \\
\hline Hypertension & 1.57 (0.96 to 2.58$)$ & 0.074 & 1.18 (0.67 to 2.06$)$ & 0.568 \\
\hline Hypercholesterolaemia & 1.16 (0.70 to 1.93$)$ & 0.569 & & 0.082 \\
\hline Diabetes & 1.46 (0.84 to 2.55$)$ & 0.184 & 1.00 (0.53 to 1.88$)$ & 0.999 \\
\hline Positive family history & 1.27 (0.77 to 2.08$)$ & 0.346 & & \\
\hline Smoking & 1.52 (0.87 to 2.65$)$ & 0.143 & 1.72 (0.94 to 3.13 ) & 0.077 \\
\hline
\end{tabular}

of 4.8 years. ${ }^{10}$ There was no difference between groups in the need for ICA and revascularisation at 5 years, which is consistent with our study. However, a lower rate of the primary endpoint (death from coronary heart disease or non-fatal myocardial infarction) was observed in the ETT plus CTCA arm. This was primarily driven by a lower rate of non-fatal myocardial infarction and the authors attribute the difference in the medical treatment which was given to ETT plus CTCA arm patients based on more correct diagnosis of CAD. Indeed, $19.4 \%$ of patients in the CTCA

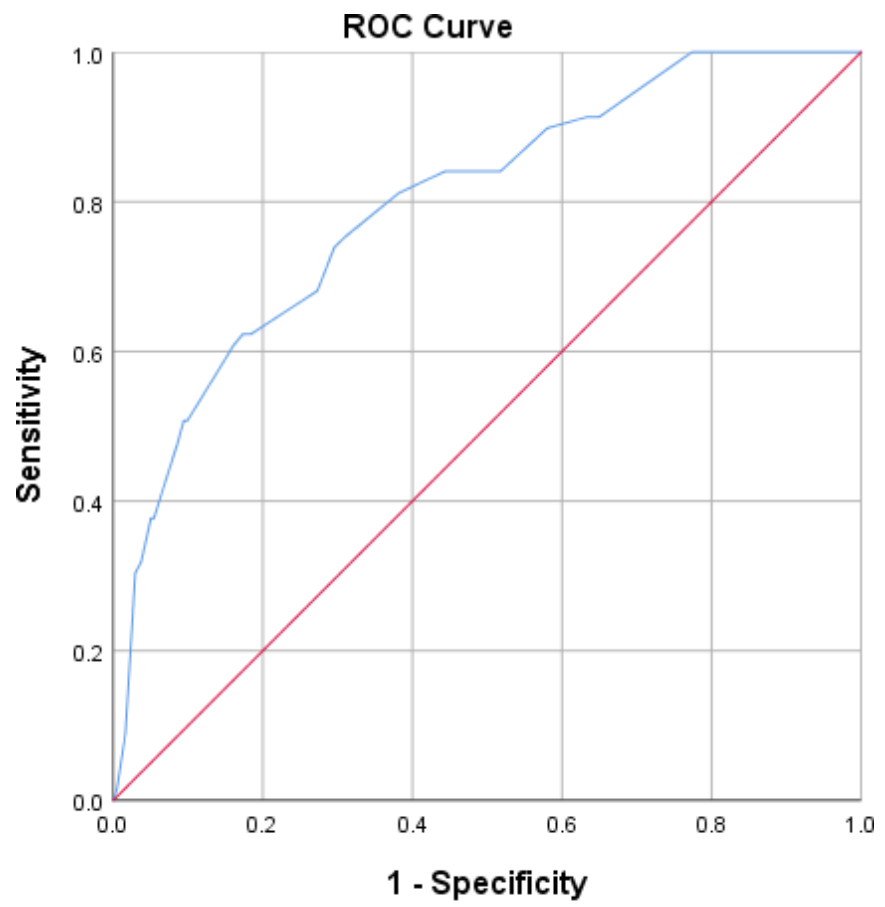

Diagonal segments are produced by ties.

Figure 3 Receiver operating characteristic (ROC) analysis for prediction of revascularisation for the European Society of Cardiology pretest probability risk score. Area under the curve $(A \cup C)=0.797(95 \% \mathrm{Cl} 0.743$ to $0.851, \mathrm{p}<0.001)$. arm commenced preventive therapy as opposed to $14.7 \%$ in the standard care arm. A difference was also noted in antianginal therapies $(13.2 \%$ vs $10.7 \%)$. The ETT, which was used as 'standard care' investigation, is known to have low sensitivity (only 50\%) in diagnosing $\mathrm{CAD}$, and ESC recommend not using it for diagnostic purposes. ${ }^{2}$ In clinical practice, it is used only when imaging modalities (functional or anatomical) are not available. In addition, the researchers compared two tests versus one test. It is not surprising that a combination of a functional plus an anatomical investigation appears to be more 'accurate' in diagnosing $\mathrm{CAD}$ compared with a functional test alone. On the other hand, SCOT-HEART highlighted the significance of diagnosing coronary atherosclerosis, which allows for primary prevention treatment. This by itself consists an important clinical implication. The functional imaging tests cannot detect haemodynamically nonsignificant coronary atherosclerosis, which undoubtedly is a weakness. On the other hand, they can detect microvascular disease which adds critical prognostic value, ${ }^{11-13}$ and this is something that anatomical tests cannot identify. Whether these differences between functional and anatomical imaging tests are clinically significant is not entirely clear based on current evidence. ${ }^{9}$

Our findings further suggest lower cost when the patients are investigated initially with SE, based on UK tariff rates. This is probably attributable to the lower cost of SE compared with CTCA, but also to the fact that in our population a lower proportion of patients required further investigation after SE compared with the need for additional testing following CTCA (2.6\% vs 4.5\%; $\mathrm{p}=0.198$ ), though the absolute numbers were relatively small. When the two strategies were compared in a lowintermediate risk population, the cost was even lower with a SE-first approach. In addition to lower cost, SE's prognostic role should not be overlooked, given that the presence of ischaemia is predictive of adverse events including unstable angina, myocardial infarction, late 
revascularisation and cardiac death. ${ }^{514-17}$ The wide availability and low cost have established SE as first-line investigation in many institutions worldwide.

The updated NICE guidelines have received a lot of criticism for removing the PTP RS and downgrading the usefulness of functional tests. ${ }^{7}$ However, NICE claim that the RS was not able to identify sufficiently patients with $<10 \%$ and $>90 \%$ PTP. $^{8}$ Hence, clinical evaluation alone was considered inadequate in distinguishing patients who need or not further investigation. On the contrary, ESC recommend the use of RS, which has been proven to have a predictive role. ${ }^{2}{ }^{3}$ In our study, ESC RS was shown to be the only independent predictor of revascularisation, suggesting that the combination of demographic and clinical variables can be useful in contemporary clinical practice to identify patients who will require coronary revascularisation.

ESC have now published new guidelines on stable coronary syndromes, ${ }^{18}$ which were not available prior to our article submission. Interestingly enough, they still recommend the use of RS and the findings of our study are in line with this recommendation. Additionally, functional tests are still recommended as first-line investigation in symptomatic patients when obstructive CAD cannot be excluded by clinical assessment alone (ie, RS). In the same population, CTCA can also be used, which is an upgrade of CTCA's role. The Task Force comment that CTCA is preferable in patients with a lower range of clinical likelihood of $\mathrm{CAD}$ and characteristics associated with good image quality. On the contrary, they mention that non-invasive functional tests have better rule-in power.

Current literature, along with our study, suggests that both CTCA and functional tests can serve equally well as first-line investigations in patients with new onset stable chest pain. Local expertise, availability and tariffs may make clinicians choose one or the other. In a period that the NHS faces significant financial difficulties, with many Trusts not having the capacity of CT scanners to accommodate the increase in CTCA requests, SE can be a very useful diagnostic tool, relieving stress on facilities and clinicians. Given that the majority of new referrals to cardiology specialist units are for new onset chest pain, we believe that our study will be of special interest to the cardiologists worldwide and may also trigger further research and discussion on the ongoing controversy between NICE and ESC guidelines.

\section{Study limitations}

This is a non-randomised observational two-centre study with the inherent limitations and bias and the results should be interpreted accordingly. Two discrete populations from two different centres were compared. The baseline characteristics and the prevalence of risk factors for $\mathrm{CAD}$ were similar in the two investigated groups. However, the symptom profile of two groups was different, which may have an impact on the results. This difference highlights the subjectivity in symptoms assessment by clinicians. In any event when the type of chest pain was included in the RS, the latter was found to be similar between the groups. In group B, $13.7 \%$ of patients were deemed unsuitable for CTCA. This percentage appears high, but it was the clinician's decision to avoid the risk of higher dose of beta-blockers and/or high-dose radiation with retrospective gating in a 64-clice CT scanner. Patients who were referred directly for ICA have been excluded from the cost analysis and this may bias the cost analysis. However, the scope of this study was to compare the noninvasive tests as gatekeepers to ICA and not the direct ICA referrals. In addition, high-risk patients are deemed appropriate to be referred directly for ICA according to both NICE and ESC guidelines. No follow-up data have been recorded regarding patient outcome as the scope of the study was to assess the frequency of ICA and revascularisation, when two different guidelines are followed and also evaluate the cost effectiveness. In the view of the absence of long-term follow-up data, the revascularisation rate in our cohort appears lower compared with other studies. Finally, the cost analysis is based on UK tariff rates.

\section{CONCLUSION}

UK NICE and ESC-recommended investigative strategies, when applied to discrete but similar populations, led to comparable rates of invasive coronary angiograms and revascularisation, but lower cost when SE was used as firstline investigation. The ESC RS was found to be the only independent predictor of revascularisation. It is reasonable to conclude that healthcare providers may follow either strategy, considering local service availability, expertise, local tariffs and budget, and PTP RS remains of clinical utility in a contemporary population.

Contributors AP collected data, performed the statistical analysis and interpretation of the results and wrote the manuscript. GFV, SJD and TA collected data and revised the manuscript. EA, TG, and ES created the registry, collected data and revised the manuscript. JB and MM revised the manuscript and provided expert opinion throughout. KAF conceived the idea for the project, revised the manuscript and provided expert opinion throughout.

Funding The authors have not declared a specific grant for this research from any funding agency in the public, commercial or not-for-profit sectors.

Competing interests None declared.

Patient consent for publication Not required.

Provenance and peer review Not commissioned; externally peer reviewed.

Data availability statement Data are available upon reasonable request. Deidentified participant data can be available upon reasonable request.

Open access This is an open access article distributed in accordance with the Creative Commons Attribution Non Commercial (CC BY-NC 4.0) license, which permits others to distribute, remix, adapt, build upon this work non-commercially, and license their derivative works on different terms, provided the original work is properly cited, appropriate credit is given, any changes made indicated, and the use is non-commercial. See: http://creativecommons.org/licenses/by-nc/4.0/.

ORCID iD

Alexandros Papachristidis http://orcid.org/0000-0002-7606-118X

\section{REFERENCES}

1 National Institute for Health and Care Excellence. Addendum to clinical guideline (CG95), Chestpain of recent onset: assessment and 
diagnosis, 2016. Available: https://www.nice.org.uk/guidance/GIDCGWAVE0774/documents/addendum

2 Task Force Members, Montalescot G, Sechtem U, et al. 2013 ESC guidelines on the management of stable coronary artery disease: the task force on the management of stable coronary artery disease of the European Society of cardiology. Eur Heart $J$ 2013:34:2949-3003.

3 Diamond GA, Forrester JS. Analysis of probability as an aid in the clinical diagnosis of coronary-artery disease. $N$ Engl $J$ Med 1979;300:1350-8.

4 Patel MR, Peterson ED, Dai D, et al. Low diagnostic yield of elective coronary angiography. N Engl J Med 2010;362:886-95.

5 Papachristidis A, Roper D, Demarco C, et al. The prognostic role of stress echocardiography in a contemporary population and the clinical significance of limited apical ischaemia. Echo Res Pract 2016;3:105-13.

6 Hecht HS, Shaw L, Chandrashekhar YS, et al. Should NICE guidelines be universally accepted for the evaluation of stable coronary disease? A debate. Eur Heart J 2019;40:ehz024 https://doi. org/

7 Cremer PC, Nissen SE. The National Institute for health and care excellence update for stable chest pain: poorly reasoned and risky for patients. Heart 2017:103:972-4.

8 Kelion AD, Nicol ED. The rationale for the Primacy of coronary CT angiography in the National Institute for health and care excellence (NICE) guideline (CG95) for the investigation of chest pain of recent onset. J Cardiovasc Comput Tomogr 2018;12:516-22.

9 Douglas PS, Hoffmann U, Patel MR, et al. Outcomes of anatomica versus functional testing for coronary artery disease. $N$ Engl J Med 2015;372:1291-300.
10 , Newby DE, Adamson PD, et al, SCOT-HEART Investigators. Coronary CT angiography and 5-year risk of myocardial infarction. $N$ Engl J Med 2018;379:924-33.

11 Cortigiani L, Rigo F, Gherardi S, et al. Additional prognostic value of coronary flow reserve in diabetic and nondiabetic patients with negative dipyridamole stress echocardiography by wall motion criteria. J Am Coll Cardiol 2007:50:1354-61.

12 Rigo F, Sicari R, Gherardi S, et al. The additive prognostic value of wall motion abnormalities and coronary flow reserve during dipyridamole stress echo. Eur Heart J 2008;29:79-88.

13 Sicari R, Nihoyannopoulos P, Evangelista A, et al. Stress echocardiography expert consensus statement: European association of echocardiography (EAE) (a registered branch of the ESC). Eur J Echocardiogr 2008;9:415-37.

14 Marwick TH, Case C, Sawada S, et al. Prediction of mortality using dobutamine echocardiography. J Am Coll Cardiol 2001;37:754-60.

15 Sicari R, Pasanisi E, Venneri L, et al. Stress echo results predict mortality: a large-scale multicenter prospective international study. $J$ Am Coll Cardiol 2003;41:589-95.

16 Picano E, Mathias W, A P, Pingitore A, et al. Safety and tolerability of dobutamine-atropine stress echocardiography: a prospective, multicentre study. The Lancet 1994;344:1190-2.

17 Picano E, Sicari R, Landi P, et al. Prognostic value of myocardial viability in medically treated patients with global left ventricular dysfunction early after an acute uncomplicated myocardial infarction: a dobutamine stress echocardiographic study. Circulation 1998;98:1078-84.

18 Knuuti J, Wijns W, Saraste A, et al. 2019 ESC guidelines for the diagnosis and management of chronic coronary syndromes. Eur Heart J 2019:pii: ehz425. 